\title{
Editorial
}

\section{Producción y distribución de cerámica en los Andes prehispánicos: perspectivas contextuales y prácticas}

El 2 de septiembre del ańo 2005 en la ciudad de Lima, se llevó a cabo la cuarta edición del simposio denominado Seminario de Arqueología UNFV, el cual llevó el título de La producción y distribución de cerámica como indicador social y politico en los Andes prehispánicos y coloniales. A pesar de lo sugestivo del título, las ponencias presentadas tuvieron una gran variedad de enfoques, desde los más «clásicos» hasta los más innovadores, pero todos concentrando los esfuerzos, obviamente, en estudios sobre cerámica. Las 15 ponencias admitidas oficialmente (dos de las cuales lamentablemente no fueron presentadas por no llegar a tiempo los conferencistas) tomaron diversos rumbos metodológicos para llegar a exponer sus resultados respectivos. Para cumplir con los requerimientos del evento y la publicación se presentaron trabajos que podían incluir tanto datos estilísticos (Ángeles, este número; Valdez, este número; Dolorier y Casas, este número; Vallejo, este número) como arqueométricos (Chapdelaine et al., este número), mientras que algunos, a partir de casos prácticos, proponen lineamientos metodológicos e interpretativos de acuerdo a realidades diversas (Vallejo, éste número; Uribe, este número). Otros casos que podemos mencionar son aquellos que tomaron más de una perspectiva para mostrar sus respectivos aportes (Cook, este número; Valdez y Valdez, este número), los cuales combinan enfoques funcionales y contextuales para llegar a las inferencias del caso. Es significativamente relevante la información proveniente de talleres, la cual nos brinda panoramas bastante amplios para entender patrones de consumo y políticas de producción (Tschauner, este número). Como se puede apreciar, tenemos una gran variedad de enfoques y tendencias metodológicas, las cuales están permitiendo llegar a niveles de inferencia óptimos.

Pasando a los casos netamente prácticos, Lidio Valdez intenta seguir manteniendo los postulados que sostienen que el valle de Acarí, durante el Período Intermedio Temprano, mantiene una autonomía política con respecto a la sociedad Nasca. En trabajos anteriores, esta hipótesis ya había sido 
esgrimida por el autor a partir del estudio de contextos funerarios, señalando una suerte de etnicidad, la cual se haría más fuerte ante las presiones externas, aunque permitiendo la entrada de ciertos elementos estilísticamente relacionados a Nasca. Se brinda un panorama inicial sobre el contexto social en el cual se desenvuelve y circula la alfarería nasca.

Claude Chapdelaine, Víctor Pimentel y Jorge Gamboa nos brindan nuevos datos en torno al panorama político que ellos interpretan como un control directo del valle de Santa por parte del área nuclear moche durante la Fase IV, que estilísticamente identifica a esta sociedad. Pero, a su vez, se propone la emergencia de una identidad «santeña» en el valle, producto de las presiones externas y otros factores internos. A partir de la identificación de sitios productores de cerámica y relevantes datos radiocarbónicos se presenta una interesante y atrevida propuesta de parte de los autores, los cuales intentan comprender de manera global la naturaleza del estado Moche Sur.

A partir de una muestra de cucharas provenientes del sitio wari de Conchopata, Anita G. Cook discute algunos patrones de consumo, circulación y uso poco conocidos para estos objetos, pues generalmente estos son asociados a contextos de índole doméstica. Ciertos contextos de alto valor ritual y simbólico contienen a estas cucharas, las cuales podrían adquirir a partir de esta situación social un significado que hasta el momento ha pasado casi desapercibido. Todo haría indicar que estas cucharas también podrían ser enmarcadas dentro ciertos eventos de carácter funerario y ritual, adquiriendo un prestigio que recién empieza a ser entendido.

Siguiendo con los trabajos centrados en el Horizonte Medio, pasamos al sitio wari de Marayniyoq en Ayacucho. Lidio Valdez y J. Ernesto Valdez nos ubican en una situación social que ha sido casi ignorada por la mayoría de investigadores que realizan estudios sobre cerámica en esta zona: la reutilización de vasijas (desechadas) por parte de estratos sociales poco favorecidos. Todo haría indicar que muchas piezas de alfarería que en algunos contextos habrían sido desechadas o descartadas, son rescatadas y reutilizadas por personas pertenecientes a una clase social de pocos recursos materiales, y con mucho menos recursos en buenas condiciones o nuevos. Los autores, a partir de esta situación, intentan demostrar la existencia de contradicciones sociales existentes entre clases, aquellas que pueden hacer derroches de poder al sacrificar cerámica fina y otra que tiene que «reciclar» desechos para satisfacer sus necesidades. Discutible enfoque, el cual expone muchas evidencias interesantes. 
Tomando como punto de referencia el valle de Asia de la costa surcentral del Perú, Rommel Ángeles nos brinda un panorama bastante amplio en torno a la distribución de los sitios que presentan cerámica del estilo Cerro del Oro. No solo contamos con un reporte de sitios que se asocian a éste estilo, sino que también este es caracterizado desde lineamientos puramente estilísticos y cronológicos, brindando una completa ubicación a partir de las evidencias con las que se cuentan, las cuales se restringen casi totalmente a material de superficie y colecciones. Ese aporte se constituye en un punto de partida para retomar la discusión en torno a la irrupción del Horizonte Medio en esta parte de la costa peruana, fenómeno que estilísticamente se revela como una materialidad bastante ecléctica y dinámica.

Retomando la costa sur peruana, Valdez nos muestra los resultados de un hallazgo fortuito en el sitio conocido como La Oroya. Un rico contexto de ofrendas del Horizonte Medio es documentado en este trabajo. Cabe aclarar que se admitió este aporte extra, el cual no fue presentado en el simposio, debido al carácter relevante de la información pues reporta de manera muy sucinta otro caso de la llamada «tradición de ofrendas» del Horizonte Medio. Si bien el trabajo es de corta extensión, éste cumple con el objetivo de informar sobre la existencia de otro caso de este tipo de eventos. Al igual que en algunos otros sitios del Horizonte Medio, en La Oroya se habrían suscitado eventos de carácter ritual dentro de los cuales el «sacrificio» de vasijas finas de diversos estilos del Horizonte Medio habrían sido una situación propiciada en determinados contextos sociales de elite.

Obteniendo información primaria y sumamente importante del taller de Pampa de Burros, Hartmut Tschauner nos lleva a entender que en la sociedad Chimú existieron unidades de producción que actuaban o trabajaban ajenas a los mandatos o lineamientos estatales. El sugestivo enunciado a través de una analogía que dice textualmente «los olleros no son del inka» no hace más que captar nuestra atención, a través de una analogía, hacia rumbos que nos lleven a comprender que no siempre los centros estatales tienen la capacidad de supervisar todo tipo de producción. Un caso interesante, el cual queda para posteriores debates y reevaluaciones.

Retomando un caso ya casi olvidado por los arqueólogos que trabajan en la costa central peruana, Camilo Dolorier y Lyda Casas realizan un análisis estilístico-iconográfico del estilo Tricolor Geométrico (Horizonte Medio Época 4) sobre la base de ajuares funerarios procedentes del sitio de Huallamarca en el valle bajo del Rímac y cruzando esta información inédita con muestras publicadas, procedentes estas últimas de Ancón y otros sitios importantes. Lo más resaltante de este estudio sería, a nuestro parecer, la ma- 
nera como se insertan los resultados para la comprensión de un fenómeno posterior: la génesis de la sociedad y el estilo Ychsma. El origen de Ychsma es una de las etapas más oscuras en el estudio de esta sociedad (Vallejo, este número). Este aporte será de utilidad para llenar parcialmente ese espacio vacío.

Continuando con los estudios en la costa central peruana, Francisco Vallejo nos brinda algunas reflexiones y discusiones, las cuales representarían una continuación de trabajos anteriores iniciados por el autor en algunos sitios ychsma del valle del Rímac. Definir unidades estilísticas, contextos, distribución y cronología relativa es el objetivo primordial de este importante aporte, el cual tiene una base de datos bastante amplia, compuesta por diversos tipos de contextos como los funerarios, los llamados "pagos» a la tierra, basurales y otros alternativos. Muchos aspectos terminológicos y estilísticos son despejados a partir de una revisión crítica de trabajos anteriores, tratando de proporcionar una visión bastante global de la sociedad Ychsma, la cual ya estamos comenzando a entender de manera más completa desde algunos años, a raíz de las múltiples investigaciones que se vienen ejecutando en los valles del Rímac y Lurín.

Dejando de lado los Andes Centrales, Mauricio Uribe nos entrega una magnífica contribución para entender cómo «El Inka» se manifiesta en el desierto de Atacama, Norte Grande de Chile. Este artículo constituye un valioso aporte para entender cómo se materializa este fenómeno en esta parte de Sudamérica, en la cual los estudios centrados en los períodos tardíos se encuentran muy avanzados. Se hacen importantes propuestas interpretativas y metodológicas para comprender cómo la cerámica y su relación con los caminos se enmarca dentro de un complejo panorama en el cual muchas sociedades adoptan diversos mecanismos políticos ante la presencia del Tawantinsuyu. A su vez, se escenifica la manera como estos sistemas sociales se prolongan hasta tiempos de la irrupción europea en el territorio. Siguiendo proporcionando alcances en torno a este tema de interés regional, Uribe nos brinda un interesante caso para entender la manera como "el Inka» se asienta sobre esta zona de Chile, y dejando en claro que la «generosidad» del imperio obedece a complejas modalidades de manejo político que se pueden entender a partir de la distribución de cerámica a nivel regional.

Habiendo hecho este breve repaso sobre los artículos presentados en esta entrega, debemos dejar en claro algunos aspectos relativos a la edición de este conjunto de artículos. Desde el mes de septiembre del año 2005, exactamente desde el día del evento mencionando líneas atrás, el suscrito 
empezó una convocatoria a diversos profesionales peruanos y extranjeros para la conformación de un grupo de asesoría editorial, el cual comenzó a trabajar desde el mes de enero del 2006. Las funciones principales de este grupo de profesionales se centraron en garantizar un control de calidad sobre los trabajos remitidos al editor, a través de críticas y comentarios sobre los mismos. En los créditos de este preámbulo figuran los nombres de estos nueve colegas que han hecho posible que estos trabajos vayan por el camino correcto en lo concerniente a niveles de exigencia. A pesar de que algunos aportes tuvieron que ser rechazados por diversos motivos, creemos que fue necesario contar con esta valiosa asesoría.

La presente publicación no hubiera sido posible sin el apoyo de mis colegas de la Universidad de Chile, Mauricio Uribe Rodríguez y Andrés Troncoso, director del Departamento de Antropología de la Universidad de Chile y editor de la Revista Chilena de Antropología respectivamente. A pesar de que casi todos los artículos refieren a zonas ubicadas dentro del Perú, ellos se mostraron muy interesados en publicar estas actas parciales del evento, en este No 20 de la revista, correspondiente al ańo 2009. A ellos gracias totales por su generosidad y comprensión.

Así también, gracias al Instituto Francés de Estudios Andinos, el presente número pertenece al Tomo 9 de la colección Actes \& Mémoires de l'Institut Français d'Études Andines. Gracias a las gestiones y aval respectivo de esta importante entidad, tenemos el honor de ser parte de esta serie.

Queremos agradecer a todos aquellos que hicieran posible el evento que dio origen a esta publicación. En primer lugar, a la Facultad de Humanidades de la Universidad Nacional Federico Villarreal (Lima, Perú), a través del entonces decano, el doctor Lorgio A. Guibovich Del Carpio, quien facilitó todos los avales respetivos para la realización del evento, sin poner ninguna objeción a que este se realice, por primera vez, fuera del claustro universitario. El suscrito formó parte de la comisión organizadora del evento, junto a Marina Romero y Claudia Arce, y contamos con la entusiasta colaboración de un grupo de apoyo de estudiantes de arqueología de las bases 2004 y 2005 de nuestra casa de estudios, entre los que se encontraron Isabel Cinthya Caja, Patricia Díaz, Lea Lorena Rojas, María Paula Velarde, Luis Alfredo Fernández Wanda, Melissa del Alcázar, Diana Inga, Luis Núnez Yaya, Audry Pallete y muchos otros que colaboraron activamente.

Nuevamente debemos agradecer al Instituto Francés de Estudios Andinos, que ha permitido la inclusión de este número de la revista en la serie mencionada líneas arriba. Al doctor Henri Godard, hasta el año 2007 direc- 
tor de esta prestigiosa entidad científica, quien gentilmente aceptó nuestra propuesta y solicitud para incluirnos en esta colección y apoyar la presentación de la publicación. Nora Araujo tuvo mucha paciencia a la hora de tramitar las gestiones respectivas. La doctora Anne-Marie Brougère, responsable de publicaciones del IFEA, se encargó de cerciorarse de que nuestros asesores editoriales trabajaran con la seriedad respetiva. Todos ellos forman un grupo profesional de primer nivel.

A Carlos Del Águila, quien durante su gestión como director del Museo Nacional de Arqueología, Antropología e Historia del Perú (MNAAHP) nos permitió usar el auditorio de esta entidad para la realización del simposio. Su apoyo y paciencia permitieron que tengamos este importante aval para nuestras actividades. Así también desde su posición como asesor de edición, hizo mucho por contribuir a elevar el nivel de algunos artículos. A Carlos nuestro más sincero reconocimiento y agradecimiento, no solo por su fructífera gestión como director del MNAAHP, cargo que lamentablemente ya no ostenta, sino también por su amistad.

El grupo de asesores editoriales mencionados anteriormente fue fundamental para elevar el nivel de las contribuciones. Entregando sus fichas editoriales dentro de los plazos fijados contribuyeron de manera decisiva a nuestra labor. Las gracias a todos los miembros de este formidable grupo. Sin ningún orden en particular, las gracias a Mauricio Uribe (Universidad de Chile, Chile), Izumi Shimada (Southern Illinois University, USA), Claude Chapdelaine (Université de Montréal, Canada), Iván Ghezzi Solís (Pontificia Universidad Católica del Perú), Carlos Del Águila Chávez (Instituto Andino de Estudios Arqueológicos - INDEA, Perú), Martti Pärssinen (University of Helsinki, Finland), Tiffiny A. Tung (Vanderbilt University, USA), Carlos Farfán (Codirector del Proyecto Ychsma de la Universidad Libre de Bruselas, Bélgica - Universidad Nacional Federico Villarreal, Perú) y Rafael Segura Llanos (Pontificia Universidad Católica del Perú). El profesionalismo y buena voluntad de cada uno de ellos es algo que ha contribuido a la presente publicación.

\section{Claudio César Olaya}

Universidad Nacional Federico Villarreal, Facultad de Humanidades, Escuela Profesional de Antropología y Arqueología, Especialidad de Arqueología 